\title{
Conservación genética
}

\section{La relación genética entre [anarias y el "enclave continental Macaronésico" vista a través de la diversidad de las especies de Androcymbium

\author{
DOI: $10.15366 /$ cv2019.23.005
}

La relación entre las Islas Canarias y el llamado "enclave continental Macaronésico" ha sido ampliamente investigada en estudios que han indicado una elevada relación florística, taxonómica y genética entre estas dos regiones (Caujapé-Castells et al., 2017; García-Verdugo et al., 2019). Sin embargo, la comparación de la variación genética entre endemismos insulares y sus congéneres continentales sigue siendo extremadamente rara, a excepción de algunos casos (García-Verdugo et al., 2017). Además, salvo en las excepciones comentadas, la mayoría de las investigaciones se caracterizan por un pobre muestreo, tanto en el número de poblaciones, como en la representatividad de la distribución de los taxones estudiados o el número de efectivos recolectados, por lo que carecemos de indicadores adecuados para interpretar los orígenes de la alta diversidad genética detectada en la flora Canaria.

El proyecto ENCLAVES (MAC/3/C 141) tuvo como objetivo ampliar desde una perspectiva multidisciplinar el conocimiento de ambas floras y cuantificar, por vez primera con datos basados en muestreos exhaustivos, el nivel y sentido del intercambio de biodiversidad vegetal entre ambas regiones, después de que investigaciones llevadas a cabo por varios grupos avalen la tesis de que muchos endemismos de las islas Canarias orientales se hallan más próximos genéticamente a sus congéneres del enclave macaronésico continental que a los de las islas Canarias occidentales (Caujapé-Castells, 2011; García-Verdugo et al., 2019). Entre los objetivos de este proyecto figuraban la estimación cuantitativa del intercambio de biodiversidad vegetal entre Canarias y Marruecos, y la evaluación de la posible contribución de la flora canaria a la composición florística del continente africano.

Una de los géneros seleccionados en este proyecto fue Androcymbium Willd. (Colchicaceae), que presenta tres taxones endémicos de las islas Canarias ( $A$. psammophilum Svent., A. hierrense A. Santos subsp. hierrense y A. hierrense A. Santos subsp. macrospermum Reifenb.) y dos especies en el "enclave continental Macaronésico" ( $A$. gramineum (Cav.) Macbride y $A$. wyssianum Beauverd \& Turrett). Las especies del género Androcymbium son geófitos adaptados a vivir en zonas áridas, con un ciclo vegetativo anual. Emergen en noviembre y florecen hacia el mes de febrero, para posteriormente perder su parte aérea y desaparecer bajo tierra, donde pasan la estación seca con las reservas acumuladas en su cormo tunicado. Esta monocotiledónea presenta flores hermafroditas autocompatibles y con capacidades de reproducción vegetativa (Hoyo, 2006).

El género Androcymbium tiene una distribución disjunta entre el norte y sur del continente africano. Así mismo, las dos especies descritas en el archipiélago canario se encuentran localizadas en islas de ambos extremos: mientras A. psammophilum se distribuye en las islas más orientales (Fuerteventura y Lanzarote), $A$. hierrense es un endemismo de las islas más occidentales (La Gomera, La Palma y El Hierro). Las dos subespecies de $A$. hierrense están catalogadas como amenazadas (EN) por la UICN, mientras que A. psammophilum es reconocida como especie vulnerable, en ambos los casos debido a la actividad humana (alteración del medio, introducción de especies, herbívoras, etc.).

En este trabajo, presentamos los primeros resultados de las estimaciones de la diversidad genética de $A$. hierrense y $A$. psammophilum junto con las de dos de las poblaciones continentales de su congénere más cercano ( $A$. gramineum) muestreadas en la costa oeste de Marruecos, y sus aplicaciones a la conservación de los endemismos canarios.

\section{¿Cómo lo hemos hecho?}

Con el fin de estimar los niveles de diversidad y relación genética entre los taxones canarios y sus congéneres del "enclave continental Macaronésico", 254 individuos pertenecientes a 14 localidades de Androcymbium psammophilum, A. hierrense subsp. hierrense, $A$. hierrense subsp. macrospermum y $A$. gramineum fueron muestreadas. Para la localización de las poblaciones naturales en Canarias se recurrió al Banco de Datos de Biodiversidad de Canarias (http://www.biodiversidadcanarias.es/atlantis/common/index.jsf), que entre otros tipos de análisis, proporciona un mapa de distribución de las especies en cuadrículas UTM de 500 × 500 m con diferentes niveles de precisión y confianza.

La información genética de estas muestras se obtuvo a partir de 10 loci de microsatélites nucleares desarrollados ad hoc para este proyecto de investigación. Los microsatélites son los marcadores moleculares que se emplean actualmente en las pruebas de paternidad o genética forense. 


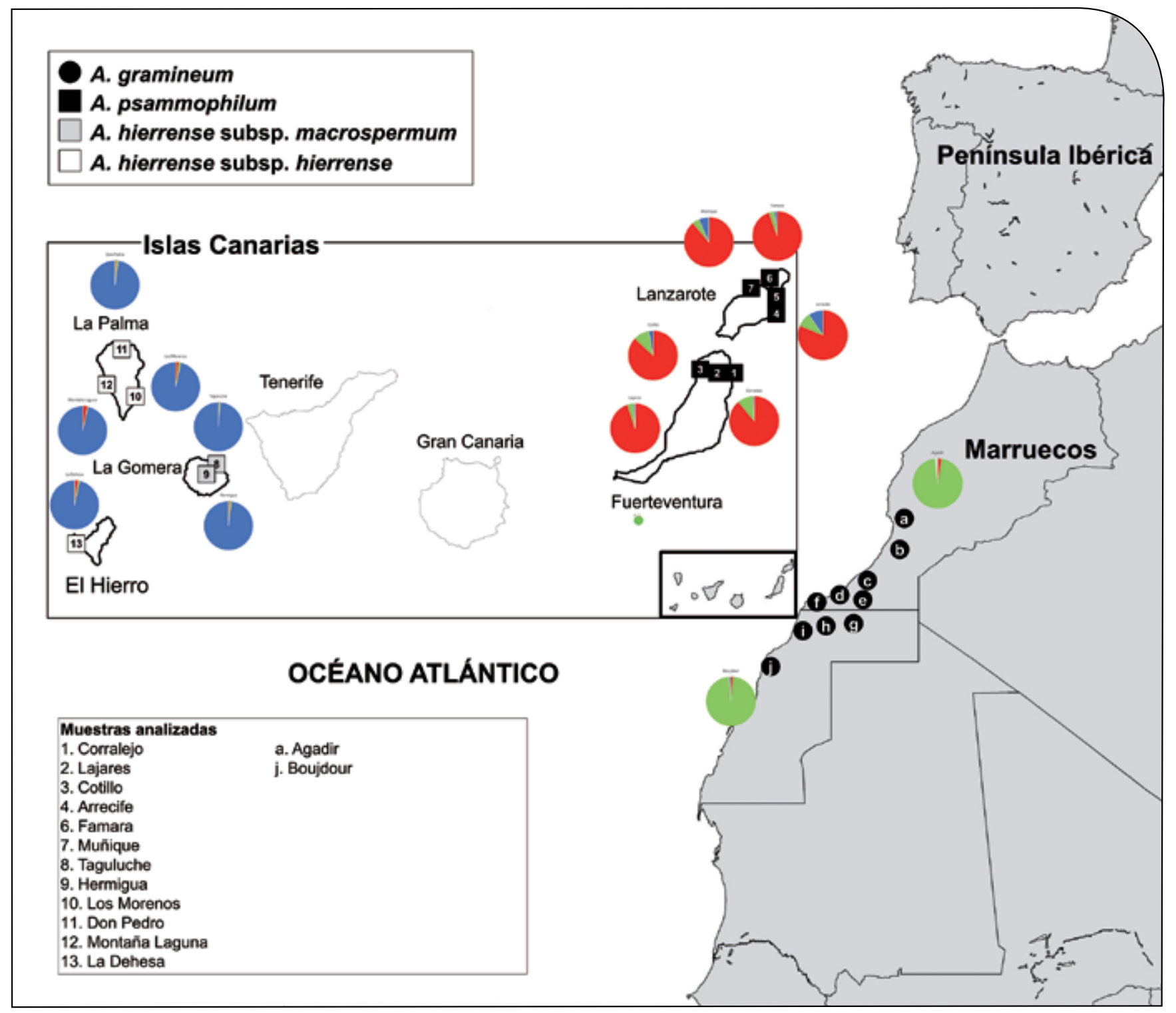

Figura 1. Localización de las poblaciones de Androcymbium muestreadas en el proyecto y asignación genética de las poblaciones analizadas en esta investigación a los grupos $(\mathrm{K}=3)$ inferidos por el análisis bayesiano.

La matriz de datos generada a partir de estos marcadores moleculares se introdujo en diferentes programas de análisis de datos genéticos. Estos nos permitieron determinar: (i) los niveles de diversidad genética mediante diferentes índices $\left(A, H_{e}, H_{o}\right)$, la diferenciación genética existente entre las poblaciones $\left(F_{S T}\right)$; (ii) cómo se distribuye esa diversidad genética dentro de cada especie (AMOVA); y (ii) la relación genética entre las poblaciones y los taxones analizados (Análisis Bayesiano y UPGMA).

\section{¿Qué nos dicen los resultados?}

Los índices de diversidad genética muestran una menor variabilidad en las islas occidentales ( $A$. hierrense, $H_{e}=0.20$ ) que en las islas orientales ( $A$. psammophilum, $\left.H_{e}=0.30\right)$, y este último valor es a su vez inferior al encontrado en las dos poblaciones analizadas de su congénere continental ( $A$. gramineum, $H_{e}=$ 0.47) (Tabla 1). Los niveles de diversidad genética detectados son en general bajos en relación con los descritos con microsatélites nucleares para otros endemismos insulares canarios (Micromeria lachnophylla, $H_{e}=0.71$, Puppo et al., 2016; llex canariensis, $H_{e}=0.53$, Sosa et al., 2013; Morella rivas-martinezii, $H_{e}=0.56$, González-Pérez et al., 2009).
El AMOVA mostró que la mayor parte de esta diversidad genética se encontraba dentro de las poblaciones tanto en $A$. psammophilum (82\%), como en $A$. hierrense (83\%), y solamente una pequeña proporción se debía a diferencias entre poblaciones (A. psammophilum: 16\%, A. hierrense: 10\%). El resto de variabilidad genética de las especies se debían a divergencia entre islas (A. psammophilum: 2\%, A. hierrense: 7\%).

El análisis de agrupamiento bayesiano (Figura 1), y el dendrograma UPGMA (Figura 2), muestran una relación más estrecha entre el endemismo de las islas orientales ( $A$. psammophilum) y su congénere continental ( $A$. gramineum), que entre el primero y el endemismo de las islas occidentales ( $A$. hierrense).

Así mismo, los datos genéticos muestran una diferencia significativa en la estructura de la variabilidad genética entre las poblaciones de las islas occidentales y orientales. En este sentido, la diferenciación genética $\left(F_{S T}\right)$ entre las poblaciones de las islas orientales es mucho menor que la que se detecta entre las poblaciones de las occidentales (Tabla 2). Las barreras geográficas que presentan actualmente las áreas de distribución del género en las islas occidentales, que en algunos casos alcanzan los $2200 \mathrm{~m}$ de altitud, dificultan o impiden el 


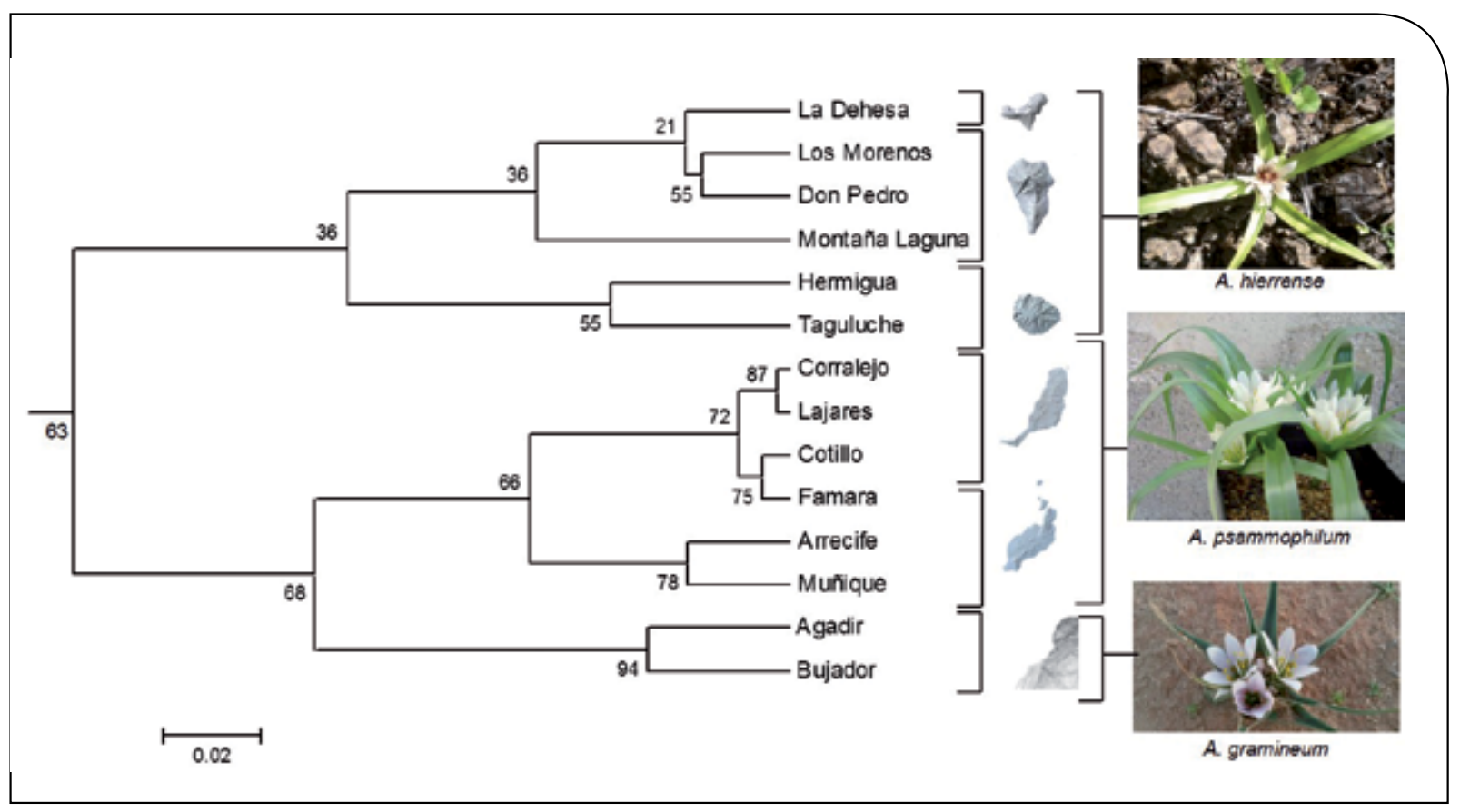

Figura 2. Dendrograma UPGMA basado en la distancia genética (Nei, 1978) entre poblaciones, incluyendo valores de bootstap, de las poblaciones de Androcymbium analizas.

flujo génico e incrementan la diferenciación genética entre poblaciones, mientras que en las islas orientales la inexistencia de accidentes montañosos significativos (en las áreas de distribución de $A$. psammophilum el relieve no supera los $220 \mathrm{~m})$, se traduce en una escasa diferenciación genética entre las poblaciones.

Estudios en curso de secuenciación de regiones plastidiales informativas (González-Pérez et al., in prep.) complementarán estos resultados con información sobre las edades y secuencias de colonización de las Islas Canarias. Estos datos, basados en los muestreos más exhaustivos realizados hasta la fecha en estas áreas de distribución del género, servirán para comprobar si Androcymbium representa otro caso de colonización reciente de las islas orientales (García-Verdugo et al., 2019), elucidar la filogeografía y secuencia de colonización insular del género, y aportar nueva luz a la posibilidad de fenómenos de retro-colonización del continente, ya apuntados en varios trabajos anteriores (Caujapé-Castells, 2011).

Estos resultados son de gran valor para establecer estrategias de conservación in situ y ex situ de los endemismos canarios. En cuanto a la conservación ex situ, los bancos de germoplasma son esenciales para la conservación de la diversidad genética. El Banco de Germoplasma del Jardín Botánico Canario "Viera y Clavijo" '- UACSIC dispone en la actualidad de una treintena de accesiones de Androcymbium que representan la distribución del género en Canarias. Los resultados genéticos aquí presentados deben tenerse en cuenta para mejorar la representatividad de la variación genética existente en las poblaciones naturales de cada taxón. La elevada fragmentación genética detectada en este trabajo dentro y entre las islas occidentales ( $A$. hierrense, Tabla 2 ), aconseja preservar semillas de todas las poblaciones conocidas. Por el contrario, en las islas orientales (A. psammophilum) detectamos una notable homogeneidad genética (Tabla 2), con lo cual un transecto en cada isla probablemente sería suficiente con el fin de representar la diversidad genética detectada.

No obstante, cualquier estrategia futura de muestreo de se- millas en estos endemismos debe tener en cuenta los análisis de las secuencias de ADN (González-Pérez et al., in prep.) y las dataciones de tiempos de divergencia, que podrían revelar subdivisiones genéticas no detectadas por los microsatélites. Las estrategias de conservación in situ pasan por la regulación de la actividad humana, especialmente en ciertas poblaciones de $A$. hierrense subsp. hierrense de La Palma y el Hierro, donde la cercanía de vertederos, de centros de tratamientos de residuos sólidos urbanos y el posible desarrollo de proyectos de urbanización amenazan seriamente a los pocos individuos existentes. En paralelo, es necesario eliminar el pastoreo en estas áreas, ya que los hábitats naturales donde se encuentran todas las poblaciones de El Hierro y La Palma son de interés comunitario (Bañares et al., 2004)

\section{Conclusiones}

- Androcymbium psammophilum parece estar genéticamente más relacionada con sus congéneres continentales (A. gramineum) que con los taxones de las islas occidentales (A. hierrense).

- Las barreras geográficas que presentan las islas occidentales actúan incrementando la diferenciación genética, mientras que en las islas orientales la inexistencia de accidentes montañosos significativos se traduce en una escasa diferenciación genética entre las poblaciones actuales.

- Los análisis genéticos muestran una clara diferenciación entre las dos subespecies de $A$. hierrense. 


\begin{tabular}{|c|c|c|c|c|c|c|}
\hline Especie & Población & N & A & AE & Ho $_{0}$ & $\mathrm{H}_{\boldsymbol{z}}$ \\
\hline \multicolumn{7}{|c|}{ A. hierrense A. Santos } \\
\hline & Don Pedro & 23.33 & 2.50 & 0 & 0.12 & 0.28 \\
\hline & Los Morenos & 32.50 & 283 & 1 & 0.03 & 0.26 \\
\hline & $\begin{array}{l}\text { Montala } \\
\text { Laguna }\end{array}$ & 8.67 & 200 & 0 & 0.21 & 0.24 \\
\hline & Hermigus & 15.33 & 200 & 0 & 0.14 & 0.20 \\
\hline & Taculuche & 7.00 & 1.33 & 0 & 0.11 & 0.13 \\
\hline & La Dehesa & 11.17 & 1.67 & 0 & 0.03 & 0.10 \\
\hline \multicolumn{2}{|l|}{ Media } & 98.00 & 2.06 & 3 & 0.12 & 0.20 \\
\hline \multicolumn{7}{|c|}{ A. ptemmoghilum Svent. } \\
\hline & Corrallejo & 10.33 & 183 & 0 & 0.19 & 0.21 \\
\hline & Cotillo & 7.17 & 2.67 & 1 & 0.32 & 0.33 \\
\hline & Lajares & 24.83 & 2.83 & 0 & 0.23 & 0.18 \\
\hline & Arrecile & 6.00 & 2.33 & 0 & 0.17 & 0.38 \\
\hline & Famara & 7.17 & 2.33 & 0 & 0.27 & 0.33 \\
\hline & Munique & 3.33 & 2.17 & 0 & 0.29 & 0.39 \\
\hline \multicolumn{2}{|l|}{ Media } & 58.83 & 2.19 & 1 & 0.24 & 0.30 \\
\hline \multicolumn{7}{|c|}{ A. gremineum (Cov)] Macbride } \\
\hline & Acadir & 9.17 & 4.00 & s & 0.42 & 0.54 \\
\hline & Boujdour & 12.83 & 3.33 & 2 & 0.42 & 0.40 \\
\hline Media & & 22.00 & 3.67 & 8 & 0.42 & 0.47 \\
\hline
\end{tabular}

Tabla 1. Relación de poblaciones analizadas y diversidad genética detectada. $N$ : tamaño de la muestra, $A$ : número medio de alelos por locus, $A E$ : número de alelos exclusivos, $H_{0}$ : heterocigosidad observada, $H_{e}$ : heterocigosidad esperada.

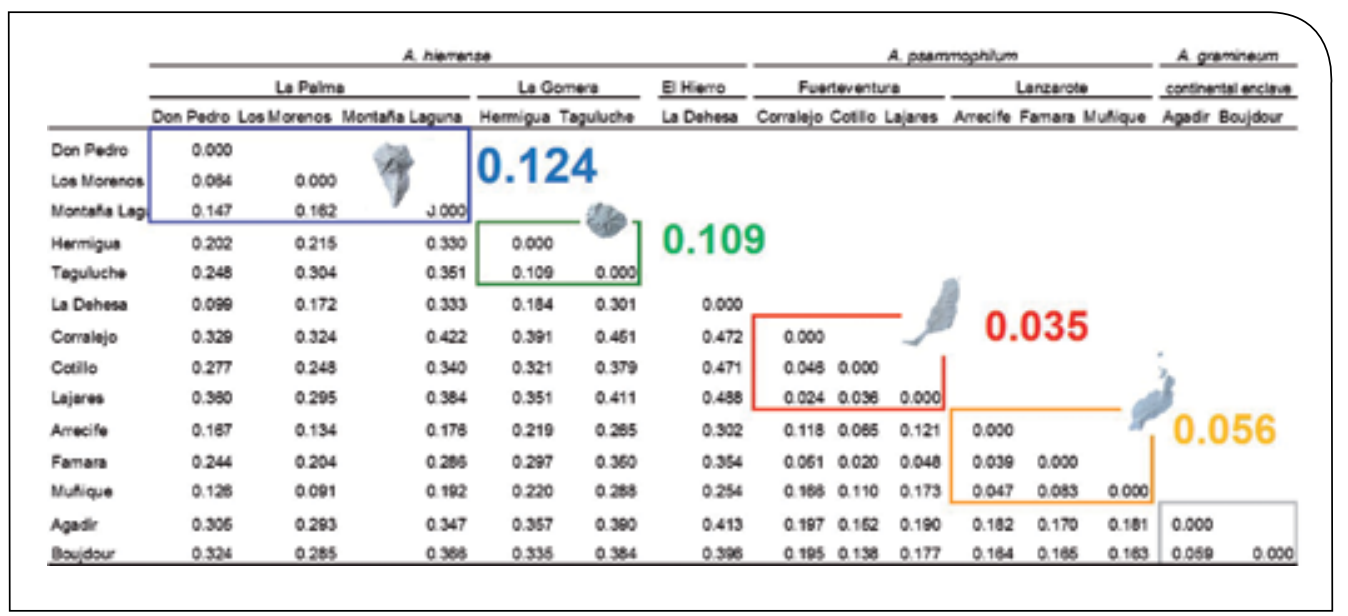

Tabla 2. Coeficiente de diferenciación genética $\left(F_{S T}\right)$ entre pares de poblaciones de Androcymbium analizadas. Se muestra el valor medio de $F_{S T}$ entre las poblaciones dentro de cada isla.

\section{Agradecimientos}

Esta investigación ha sido financiada por el Programa de Cooperación Transnacional Madeira-Azores-Canarias (PCT-MAC 2007/2013) dentro del proyecto ENCLAVES (MAC/3/C 141).

MIGUEL ÁNGEL GONZÁLEZ-PÉREZ ${ }^{1,2}$, ÁGUEDO MARRERO RODRÍGUEZ³ Y JULI CAUJAPÉ-CASTELLS' 1Departamento de Biodiversidad Molecular y Banco de ADN. Jardín Botánico Canario "Viera y Clavijo - Unidad Asociada CSIC. Cabildo de Gran Canaria. Camino del palmeral 15, 35017 Las Palmas de Gran Canaria, España. Banco de Germoplasma. Jardín Botánico Canario "Viera y Clavijo - UACSIC. Cabildo de Gran Canaria. ${ }^{3}$ Herbario LPA. Jardín Botánico Canario "Viera y Clavijo - UACSIC. Cabildo de Gran Canaria.

\section{Bibliografía}

- Bañares, Á., G. Blanca, J. Güemes, J.C. Moreno \& S. Ortiz, eds. (2004). Atlas y Libro Rojo de la Flora Vascular Amenazada de España Taxones prioritarios. Dirección General de Conservación de la $\mathrm{Na}$ turaleza. Madrid, $1.069 \mathrm{pp}$

- Caujapé-Castells, J. (2011). Jesters, red queens, boomerangs and surfers: a molecular outlook on the Canarian endemic flora. In: Bramwell D, Caujapé-Castells J (Eds.) The biology of island floras. Cambridge University Press, Cambridge, pp. 284324.

- Caujapé-Castells, J., C. García-Verdugo, A. Marrero-Rodríguez, J.M. Fernández-Palacios, D.J. Crawford \& M.E. Mort (2017). Island ontogenies, syngameons, and the origins of genetic diversity in the Canarian flora. Perspectives in Plant Evolution, Ecology and Systematics 27: 9-22.
- García-Verdugo, C., M. Mairal, P. Monroy, M. Sajeva \& J. Caujapé-Castells (2017). The loss of dispersal on islands hypothesis revisited: Implementing phylogeography to investigate evolution of dispersal traits in Periploca (Apocynaceae). Journal of persal traits in Periploca Apocyn
Biogeography 44: 2595-2606.

- García-Verdugo C. J. Caujapé-Castells, J.C. Illera M. Mairal, J. Patiño, A. Reyes-Betancort \& S. Scholz (2019). Pleistocene extinctions as drivers of biogeographical patterns on the easternmost Canary Islands. Journal of Biogeography 46: 845-859.

- González-Pérez, M.A., P.A. Sosa, E. Rivero, E.A González-González \& A. Naranjo (2009). Molecular markers reveal no genetic differentiation between Myrica rivas-martinezii and M. faya (Myricaceae). Annals of Botany 103:79-86.
- Hoyo, A. (2006). Filogenia y genética poblacional del género Androcymbium (Colchicaceae). Tesis Doctoral Universidad de Girona.

- Puppo, P., M. Curto \& H. Meimberg (2016). Genetic structure of Micromeria (Lamiaceae) in Tenerife, the imprint of geological history and hybridization on within-island diversification. Ecology and Evolution 6: 3443-3460.

- Sosa, P.A., E.A. González-González, M.A. González-Pérez \& P.L. Pérez de Paz (2013). Contrasting patterns of genetic differentiation in Macaronesian lineages of Ilex (Aquifoliaceae). Botanical Journal of the Linnean Society 173: 258-268. 